\section{NIH's blueprint for translation}

\section{By Lev Osherovich, Senior Writer}

In 2009, the NIH established a grant program, the Blueprint Neurotherapeutics Network, to aid the development of neurology therapeutics. That program has now announced its first awards to academic researchers and one company-Trevena Inc. - that have compounds or scaffolds for neurological disorders including amyotrophic lateral sclerosis, Alzheimer's disease, depression, hearing loss, age-related macular degeneration, optic neuropathy and stroke.

$\mathrm{BNN}$ is a component of the NIH's Blueprint for Neuroscience Research, "a consortium of $16 \mathrm{NIH}$ institutes and centers that put dollars from their budget into a common pot of money" to fund early stage drug development, said Rebecca Farkas, program director of the National Institute of Neurological Disorders and Stroke (NINDS).

The Blueprint consortium came together in 2004 with a series of programs focused on training neuroscientists and building tools for the neuroscience research community such as the Non-Human Primate Brain Atlas. In 2009, the consortium launched a second round of programs including Grand Challenge Explorations grants in brain imaging and pain.

Farkas and NINDS director Story Landis said BNN patches a hole in the translational research funding chain between early stage discovery work, which has been traditionally funded through small, hands-off grants such as Small Business Innovation Research (SBIR) grants, and more clinically focused initiatives, like the National Center for Advancing Translational Sciences that has been proposed by NIH director Francis Collins. ${ }^{1}$

According to Landis, BNN aims to turn hit- or lead-stage compounds identified primarily in academic labs into preclinical drug candidates. Compounds from the private sector also are eligible for BNN support.

"Academics may have promising ideas but don't have the access to the kinds of resources that pharmas have, so we're establishing contracts for medicinal chemistry services" and other components of preclinical development, said Landis.

Farkas added: "The program is designed to bring projects through medicinal chemistry optimization to Phase I. Most of the small molecules coming into this program will need further optimization to improve their pharmacokinetic properties."

In total, Trevena and 6 academic teams will receive $\$ 1.8$ million in seed grants this year to finance assay development and biological testing of compounds. The 7 teams are collectively eligible for an additional $\$ 5.2$ million over the next 5 years.

"We're providing access to millions of dollars of medicinal chemistry and consulting services" from six individuals, all of whom have experience in research roles at big pharma, said Landis. "If your project gets funded, we have the resources to push your promising therapeutics through Phase I development."

Of the six, two are individual consultants, three are presidents at biotech and pharma consulting companies and one is CSO at a scientific instrumentation and assay development company.

Indeed, the big winner is chemistry services provider Albany Molecular Research Inc., which will receive up to $\$ 43$ million over the next 5 years for compound screening and optimization for the 7 projects.

BNN hopes to advance compounds for which there is already proof of principle in animals, Farkas told SciBX.

Table 1. NIH Blueprint Neurotherapeutics Network awards.

\begin{tabular}{lll}
\hline Indication & Project description & Institution \\
\hline Amyotrophic lateral sclerosis (ALS) & Small molecule modulators of the glutamate transporter & Brigham and Women's Hospital \\
\hline Alzheimer's disease (AD) & Optimization of soluble $\gamma$-secretase modulators & University of California, San Diego \\
\hline Depression & Optimization of a new opioid receptor $\delta 1$ (OPRD1; DOR) biased agonist & Trevena Inc. \\
\hline Hearing loss & Drug discovery for the prevention of hearing loss & University of Washington \\
\hline Age-related macular degeneration (AMD) & Small molecule therapeutics for dry AMD & Columbia University \\
\hline Optic neuropathy & Triazine compounds for optic nerve regeneration & University of Miami \\
\hline Stroke & $\begin{array}{l}\text { Prostaglandin E } \mathrm{F}_{2} \text { receptor EP2 subtype (prostanoid EP2 receptor; PTGER2) } \\
\text { modulators for brain hemorrhage }\end{array}$ & Emory University
\end{tabular}




\section{Behind DOR number one}

The seven projects span a range of familiar and new molecular approaches to neurological diseases (see Table 1, "NIH Blueprint Neurotherapeutics Network awards").

At least two of the projects-one from Trevena and the other from the University of California, San Diego-are already in lead optimization.

For Trevena, the BNN grant is an opportunity to try out its biased ligand technology in a new therapeutic area. The company will get $\$ 460,000$ to optimize its depression candidate, a biased ligand for opioid receptor $\delta 1$ (OPRD1; DOR).

Biased ligands are small molecules that affect some signaling pathways downstream of a receptor without influencing other pathways that would ordinarily be activated by ligand binding.

Maxine Gowen, president and CEO of Trevena, told SciBX the grant builds on prior Blueprint-funded work with DOR that led to the discovery of a hit-stage compound. The new funding will help the company turn that hit into a clinical lead.

"Our approach is to very selectively turn on the signaling of receptors using so-called biased ligands," said Gowen. "We had a Grand Challenge grant that let us study a number of different receptors including DOR to find biased ligands. We focused on DOR for the Blueprint award because NIH had funded our earlier work" and was already familiar with Trevena's approach.

Trevena's lead candidate is TRV120027, an angiotensin II type 1 receptor (AGTR1) biased ligand that is in Phase IIa testing for acute heart failure (AHF).

Gowen said the grant will allow Trevena to apply its molecular approach in a therapeutic area outside the company's core interests of analgesia and heart disease.

She added that financing medicinal chemistry with public funds helps derisk that phase of development for venture investors.

From VCs, "you can get discovery funding and clinical funding, but the goal of the Blueprint awards is to fund work in the middle," said Gowen. "They're funding a part of the process that is quite tough to finance otherwise."

UCSD project scientist Steven Wagner received $\$ 245,000$ to optimize a new class of $\gamma$-secretase modulators for Alzheimer's disease (AD), a project that builds on his prior experience as CSO of TorreyPines Therapeutics Inc.

TorreyPines sold its $\gamma$-secretase program to NeuroGenetic Pharmaceuticals Inc. when the former company reverse-merged with Raptor Pharmaceutical Corp. in 2009.

Since then, Wagner has identified new compounds that fall under separate patents held by UCSD. Wagner wants to optimize his compounds using assays he helped develop at TorreyPines.
$\gamma$-Secretase, which cleaves amyloid precursor protein (APP) to create toxic $\beta$-amyloid $(A \beta)$ fragments, is a challenging $\mathrm{AD}$ target that is out of favor with pharmas because of safety problems with earlier compounds.

Outright inhibitors such as Eli Lilly and Co.'s semagacestat have proven too toxic, and only a handful of companies are pursuing next-generation $\gamma$-secretase modulators, which have a more subtle effect on the enzyme's activity.

Wagner hopes to find compounds that reduce the production of the $\mathrm{AD}$-associated $\mathrm{A} \beta 42$ fragment without compromising the processing of other $\gamma$-secretase substrates needed for normal neurological functions.

"We have about 1,200 compounds that modulate $\gamma$-secretase," said Wagner. "We created a class of molecules with better aqueous solubility than previous compounds."

Wagner will work with Albany Molecular to select lead candidates from this compound series and optimize their pharmacological properties.

"The hope is to make 400 analogs of the lead compound" and test them for efficacy in mouse models of $\mathrm{AD}$, said Wagner.

\section{Cycling}

BNN plans to run at least two more grant cycles, with applications for the next round due in December.

After all of the grant cycles, "the goal is to have 20 different projects come in at the top of the funnel and have one or two come out" into a clinical pipeline, said Farkas. "If a project doesn't meet its milestones, it drops out."

Milestones will include favorable pharmacokinetic and pharmacodynamic profiles, toxicology and efficacy in animals.

Investigators and grantee companies will retain rights to all IP coming from Blueprint-funded work.

Osherovich, L. SciBX 4(35); doi:10.1038/scibx.2011.980

Published online Sept. 8, 2011

\section{REFERENCES}

1. Usdin, S. SciBX 3(48); doi:10.1038/scibx.2010.1430

\section{COMPANIES AND INSTITUTIONS MENTIONED}

Albany Molecular Research Inc. (NASDAQ:AMRI), Albany, N.Y. Blueprint Neurotherapeutics Network, Bethesda, Md.

Eli Lilly and Co. (NYSE:LLY), Indianapolis, Ind. National Institute of Neurological Disorders and Stroke, Bethesda, Md.

National Institutes of Health, Bethesda, Md.

Raptor Pharmaceutical Corp. (NASDAQ:RPTP), Novato, Calif.

Trevena Inc., King of Prussia, Pa.

University of California, San Diego, La Jolla, Calif. 